\title{
Thermal Infrared Sensor (TIRS) Instrument Thermal Subsystem Design and Lessons Learned
}

\author{
Veronica Otero ${ }^{1}$ and Carol Mosier ${ }^{2}$ \\ NASA Goddard Space Flight Center, Greenbelt MD 20771 \\ and \\ David Neuberger ${ }^{3}$ \\ Edge Space Systems, PO Box 310, Glenelg, MD 21737
}

\begin{abstract}
The Thermal Infrared Sensor (TIRS) is one of two instruments on the Landsat Data Continuity Mission (LDCM), which is scheduled to launch in February of 2013. The TIRS instrument was officially added to the mission later in the flow, which led to a highly aggressive schedule that became one of the main drivers during instrument development. The thermal subsystem design of the TIRS Sensor Unit is comprised of five thermal zones which range in temperature from less than 43 Kelvin to 330 Kelvin. Most zones are proportional heater controlled, and all are within a volume of 35 cu.ft. A two-stage cryocooler is used to cool the "cold stage" including three QWIP detectors to less than $\mathbf{4 3}$ Kelvin, and cool the "warm stage" to 105 Kelvin. The excess power dissipation from the cryocooler is rejected via ammonia transport heat pipes to a dedicated Cryocooler Radiator with embedded ammonia heat pipes. The cryogenic subsystem includes a series of shells used to radiatively and conductively isolate the cold stage from the warmer surroundings. The Optical System (telescope) is passively cooled to 180-190 Kelvin using a "thermal link" (comprised of a Flexible Conductive Thermal Strap and an APG Bar) which couples the telescope stage to a dedicated radiator with embedded ethane heat pipes. The Scene Select Mechanism, which is responsible for moving the Scene Select Mirror to three distinct positions (including Nadir, Space, and On-board Black Body Calibrator pointing), runs nominally at 278 Kelvin and is thermally isolated from the cryogenic thermal zones. The Onboard Black Body Calibrator requires a dedicated radiator which allows for a temperature range of 260-330 Kelvin at the Source. The detectors are powered by the FPE Box, which is mounted to the nadir external surface of the composite honeycomb structure. There are two additional electronics boxes which are wet-mounted directly to the spacecraft shear panel, the Main Electronics Box and Cryocooler Electronics Box; thermal control of these boxes is the responsibility of Orbital Sciences Corporation, the spacecraft developer. The TIRS thermal subsystem design was successfully verified during months of testing campaign, from component \& subsystem level to two instrument-level thermal vacuum tests. The Instrument, despite an aggressive schedule, was delivered to the spacecraft vendor in February of 2012 and is currently undergoing the final stages of spacecraft environmental testing in preparation for launch.
\end{abstract}

$\begin{array}{llll}\text { GSFC } & =\text { Goddard Space Flight Center } & F C L & =\text { Flexible Conductive Link } \\ N A S A & =\text { National Aeronautics and Space Administration } & M C E & =\text { Mechanisms Control Electronics } \\ L D C M & =\text { Landsat Data Continuity Mission } & E T U & =\text { Engineering Test Unit } \\ T I R S & =\text { Thermal InfraRed Sensor } & A P G & =\text { Annealed Pyrolytic Graphite } \\ O L I & =\text { Operational Land Imager } & T I S & =\text { Telescope Isolation System } \\ F P E & =\text { Focal Plane Electronics } & F I S & =\text { FPA Isolation System } \\ M E B & =\text { Main Electronics Box } & G S E & =\text { Ground Support Equipment }\end{array}$

${ }^{1}$ Associate Branch Head, GSFC-Code 545.0, Greenbelt, MD 20771

${ }^{2}$ Senior Thermal Engineer, GSFC-Code 545.0, Greenbelt, MD 20771

${ }^{3}$ Thermal Engineer, Edge Space Systems, PO Box 310, Glenelg, MD 21737

1

American Institute of Aeronautics and Astronautics 
CCE $\quad=$ CryoCooler Electronics

SSM $=$ Scene Select Mechanism

QWIP $=$ Quantum Well Infrared Photodetectors
$W O A=$ Work Order Authorization

$F P A=$ Focal Plan Arrays

$F P W=$ Flexible Printed Wire

\section{Introduction}

$\mathrm{T}$ he Thermal InfraRed Sensor (TIRS) is one of two instruments on the Landsat Data Continuity Mission (LDCM) that launched on Feb 11, 2013 out of Vandenberg Air Force Base, CA. The primary instrument for the mission is the Operational Land Imager (OLI), built by Ball Aerospace in Boulder, Colorado. The secondary instrument, TIRS, was built at Goddard Space Flight Center (GSFC) in just over three and a half years.

In order to meet the launch date, a very aggressive schedule was implemented, which was the primary driver during instrument development. It led to the early procurement of long lead items, limiting design options early on; parallel testing of multiple components/subsystems in a short period of time; and a large project team with multiple systems engineers to help coordinate activities. The thermal team was a successful example of a highly organized subsystem that was able to maintain cost and schedule budgets throughout the development phase. A "divide and conquer" approach was used where team members were responsible for specific tasks, and weekly meetings as well as a comprehensive "To Do List" maintained the team well-informed of the ongoing activities and focused on the correct priorities. And along this demanding journey there were many lessons learned by the thermal team, which are described in the sections that follow.

\section{TIRS Instrument Thermal Zones}

The TIRS thermal design makes use of various thermal control techniques, both passive and active, in order to adequately maintain the instrument within levied requirements. The major pieces of hardware include dedicated subsystem radiators, Multi-Layer Insulation (MLI) blanketing, a large Earthshield, a two-stage cryocooler, coatings, isolating and conductive materials, heat pipes, heaters, sensors, and thermostats. The TIRS instrument is divided into two main assemblies, a Sensor Unit which sits on the spacecraft deck, and two remote electronics boxes, MEB and CCE. The Sensor Unit thermal design is the responsibility of the TIRS GSFC thermal team; the MEB and CCE external thermal design is the responsibility of the spacecraft vendor since these boxes are mounted directly to a spacecraft shear panel.

The TIRS Sensor Unit can be visualized as a series of thermal zones, which have very distinct temperature ranges, as shown in Fig. 2. The coldest zone is the Focal Plane Array held at a temperature range of 37-43 K. The Warm Stage, an intermediate cryocooler zone, is typically maintained in the 100-110 K range. The telescope zone is in the 180-190 K range. The warmer end of the instrument includes the Scene Select Mechanism (SSM) at 273293K, the Focal Plane Electronics (FPE) at 273-293 K, and Blackbody Calibrator controlled to a range of 270-320 $\mathrm{K}$. Additional subsystems in the warmer zone include the structure, cryocooler subsystem, and MEB/CCE electronics boxes. Finally, the Earthshield has a large temperature range to accommodate wide temperature fluctuations on orbit. 


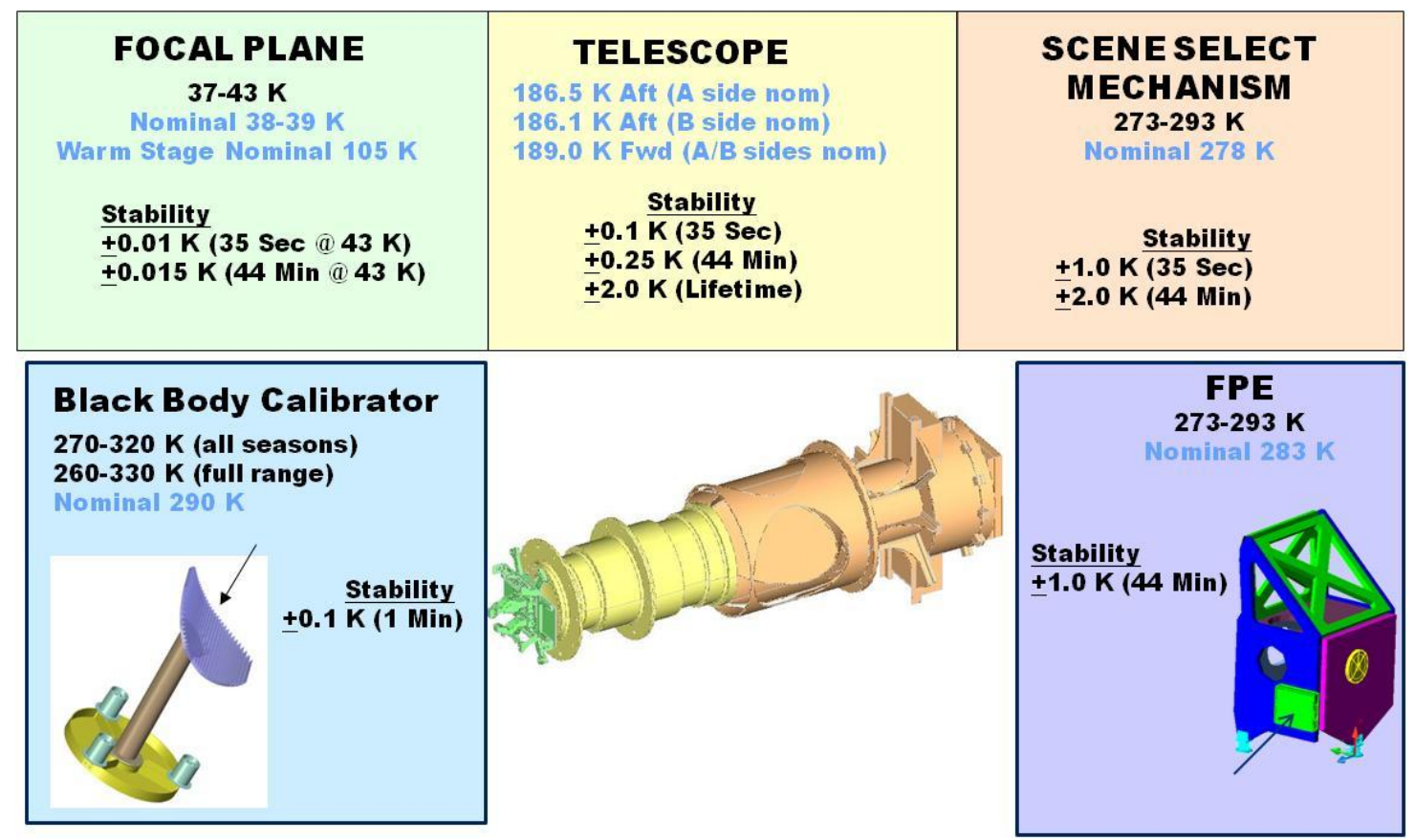

Figure 2. TIRS Sensor Unit Temperature Ranges and Stability Requirements. There are several thermal zones within the TIRS Sensor Unit, required stability and temperature ranges as shown.

\section{Cryogenic Cold Stage}

\section{A. Overview of Subsystem}

The cold stage is comprised of the following main components:

1) Three (3) Quantum Well Infrared Photodetectors (QWIPs)

2) Three (3) filters and filter holder

3) Invar baseplate to support the detectors, cable brackets, and PCBs

4) Flexible Conductive Link (FCL) made of high purity aluminum with a single layer VDA overwrap

5) Cold stage shield (shown in brown) for radiative isolation to the warm stage shield.

The detectors are maintained below 43 $\mathrm{K}$ in order to meet science requirements on orbit. There are a total of eight (8) sensors on the Focal Plane Assembly, including a combination of silicone diodes and transistors, some that are accurate to 0.05 $\mathrm{K}$ at a temperature range of $35-55 \mathrm{~K}$. The cold stage is controlled with the use of the cold stage of a two-stage cryocooler

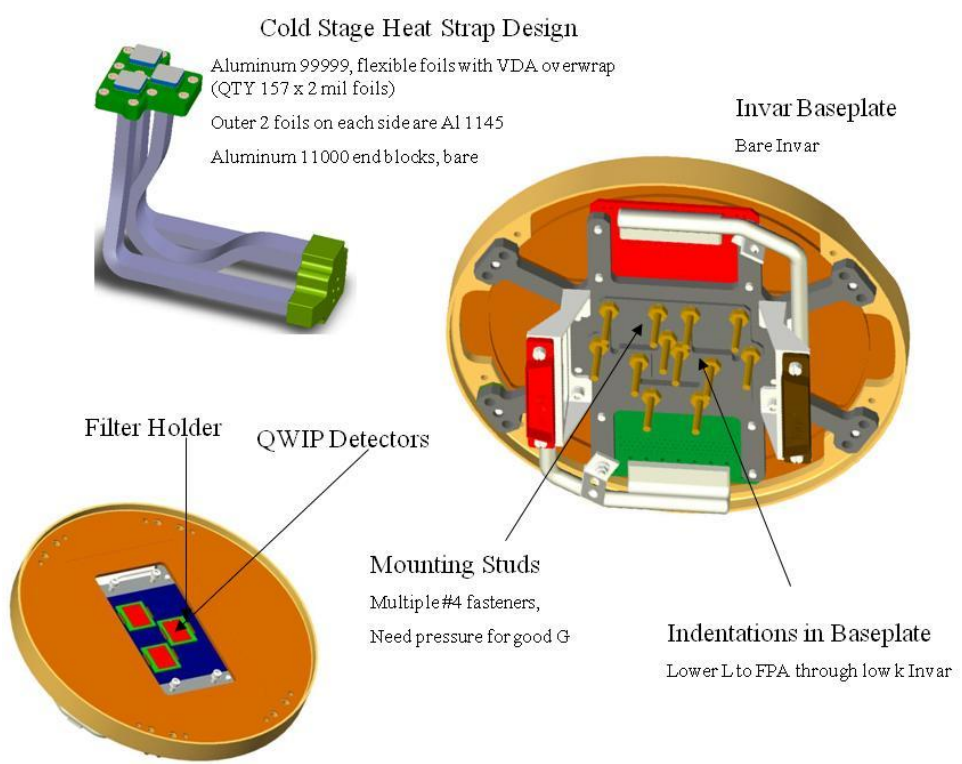

Figure 3. TIRS Cryogenic Cold Stage. The cold stage is comprised of three QWIP detectors, filters, an invar baseplate holding the subsystem together and a FCL connecting the subsystem to the cryocooler cold tip.

located a few inches away, and connected via a FCL. 
The primary heat path for the cold stage begins at the three QWIP detectors, that dissipate a total of 0.415 Watts, and ends in the Cryocooler cold stage cold tip, which is maintained stable throughout the mission. A detailed description of the heat path is as follows:

9) Three QWIPs conduct to a Silicone substrate through 0.0008 ” thick epoxy

8) Silicone substrate has a thickness of 0.020 "

7) Silicone substrate conducts to an Invar baseplate through 0.003 " thick epoxy

6) Invar baseplate has a thickness of 0.125 "

5) Invar baseplate conducts to a Sapphire isolator thru 2 mil thick indium foil

4) Sapphire electrical isolator has a thickness of 0.26 "

3) Sapphire conducts to FCL aluminum end block (warm end) thru 2 mil thick indium foil

2) FCL is a three-section design (two Al 1100 end blocks and three high purity Al flexible straps that are swaged into the two end blocks)

1) FCL end block (cold end) conducts to the cryocooler cold stage cold tip thru 2 mil thick indium foil *Conduction parasitics are introduced at various locations, mainly thru the harnessing routed to the FPE

* Radiation parasitics are introduced along the path from the surrounding warm stage (described in section IV)

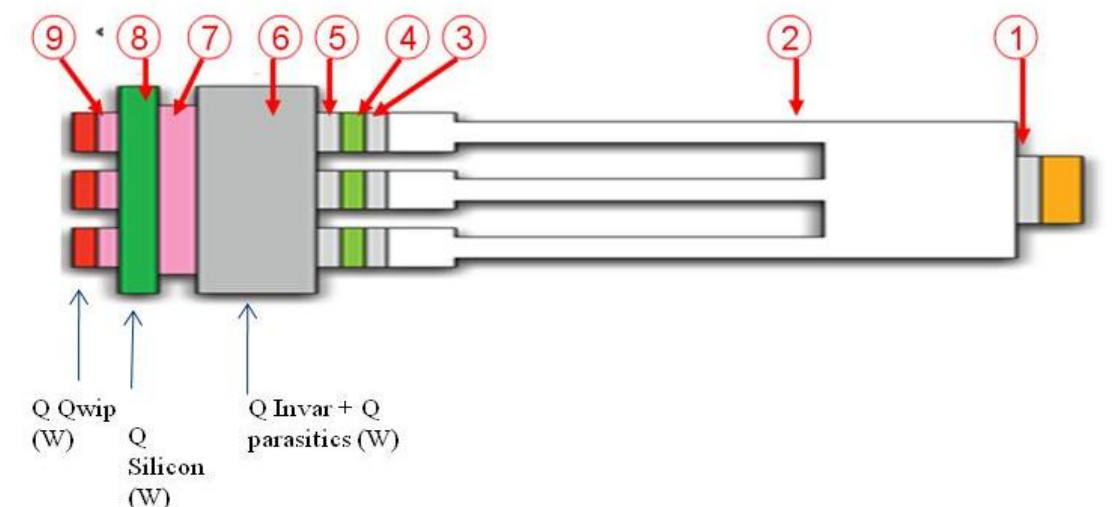

Figure 4. TIRS Cold Stage Heat Path. The heat path for the cold stage begins at the three QWIP detectors and ends at the cryocooler cold stage cold tip.

\section{B. Design Drivers and Lessons Learned}

Primary Design Driver: Minimize heat parasitics into the cold stage while maximizing conduction to the cryocooler cold stage cold tip in order to maintain the QWIP detectors below $43 \mathrm{~K}$.

Lesson Learned: Investigate the entire stackup to reduce thermal resistance. Original testing showed a larger than expected temperature rise between source and sink. The culprit was not enough epoxy being used between the silicon substrate and the invar baseplate (item 7 above). The $\%$ area of coverage was increased in the next build and the resistance was lowered.

Lesson Learned: Good material selection at the proper working temperature. Match the conductive properties to the expected temperature range. High conduction where needed and high isolation where needed. By working with the FCL vendor we used very highly conductive pure aluminum (99999) for the foils and fairly high conductive Aluminum 1100 for the end blocks. At higher temperatures one can use less pure materials since the conductive effect is not as great. The electrical isolation was provided by the sapphire (item 4) sandwiched between the baseplate and the FCL. This material gives the needed electrical isolation but has incredibly high thermal conductivity at the working temperature. Also the silicone layer (item 8) has very high thermal conductivity at these working temperatures.

\section{Cryogenic Warm Stage}

\section{A. Overview of Subsystem}

The TIRS warm stage is used to provide protection to the cold stage from the warmer surrounding instrument environment. The most challenging aspect of the design is that the cold stage must somehow be physically supported but not swamped with parasitic heat. All energy from warmer surroundings must be minimized through proper conductive and radiative isolation. The assembly of shields, isolators and shells is referred to as the FPA 
Isolation System (FIS). A system of recumbent G-10 cylinders is used to lengthen the conductive path to the warmer telescope stage, thus providing conductive isolation and a strong stable mechanical path. Each cylinder is epoxied to a metallic ring on each end (aluminum except invar used at the coldest FPA area for CTE matching). The inner cylinder is nested inside of the outer cylinder. The intersection of the rings provides the purpose of an intermediate fold ring to effectively double the length of a single cylinder. Use of low emittance coatings of gold plating on metal and single layer VDA/Kapton on G-10 reduce the radiative parasitics as well. Two shields are placed above the FPA to protect the QWIP detectors radiatively from the warmer surroundings of the optical assembly (TIRS telescope). The shields are all gold plated to provide low emissivity surfaces; except surfaces in the vicinity of the optical path necessitating the use of a black coating, Z307. The cold stage shield picks off radiation from the warm stage shield and ties into the invar cold ring of the FIS. A warm stage shield meets up at the fold rings to pick off radiative parasitics above the assembly from the telescope. Finally a thicker shield referred to as the inner cryo shell meets the fold ring below the assembly to pick off parasitics from the $180 \mathrm{~K}$ telescope stage and conductively tie the FIS to the $105 \mathrm{~K}$ warm stage sink of the cryocooler. Indium foil is used in a few of the interfaces to fill in any gaps and provide a better conductive path between components. In the majority of interfaces gold plating on the mating surfaces provides good interface conductance. Refer to Fig. 5 for a visual representation of this intricate system.

This stage is tied to the cryocooler warm stage cold tip via a FCL made of copper 101 braids held together with aluminum 1100 end blocks, allowing for cooling capability. The copper braids provide multi dimensional flexibility and high conductivity with Aluminum end blocks to save on mass. Operational heaters $(\mathrm{OP} 1 \mathrm{~A} / \mathrm{B})$ mounted at the heat strap end are used to control the warm stage to a precise temperature within the desired $100 \mathrm{~K}$ to $110 \mathrm{~K}$ temperature range, nominally $105 \mathrm{~K}$.

\section{B. Design Drivers and Lessons Learned}

Primary Design Driver: To act as an interceptor to protect cold stage from radiative and conductive heat leaks. Also serves to optimize cryocooler performance by providing a stable known temperature within a desired range.

Lesson Learned: Use proven design concepts. Using a proven design concept of isolation shells and shields for the warm stage was essential given the time constraints faced by the TIRS design teams.

Lesson Learned: Good interface conductance. Study all joints to ensure that there exists maximum thermal conductance between parts. All the good work of proper materials and thicknesses can be wasted if the interfaces between those parts are poor. Surfaces are to be reasonably smooth and flat. The interface material should be pliable and have high thermal conductance at the expected temperature range. This was accomplished in two ways: (1) both interfacing surfaces are gold plated or (2) neither interfacing surface is gold plated and an interstitial layer of indium is used.

Lesson Learned: Good radiative isolation. Use of gold plating or vapor deposition to reduce the emittance of metallic surfaces and single layer VDA/Kapton of non metallic surfaces gives the lowest possible emittances. The single layer VDA instead of MLI in several regions saved space, assembly time, and money while also removing the moisture trapping problem associated with MLI. This was used on several internal areas including FIS, TIS, telescope, and FPWs. Grounding was accomplished by using a small amount of electrically conductive epoxy between the VDA layer and the metal rings of the FIS and TIS.

Lesson Learned: Verify cryogenic system designs early. The project supported taking valuable "team time" for the fabrication, assembly, and tvac testing of a flight-like Cryo Subsystem engineering test unit (ETU) to validate the design early in the development flow. Furthermore, this allowed the mechanical team to develop a detailed assembly process and validate access, as well as provide the harness and blanket teams the ability to design and work on actual hardware. 


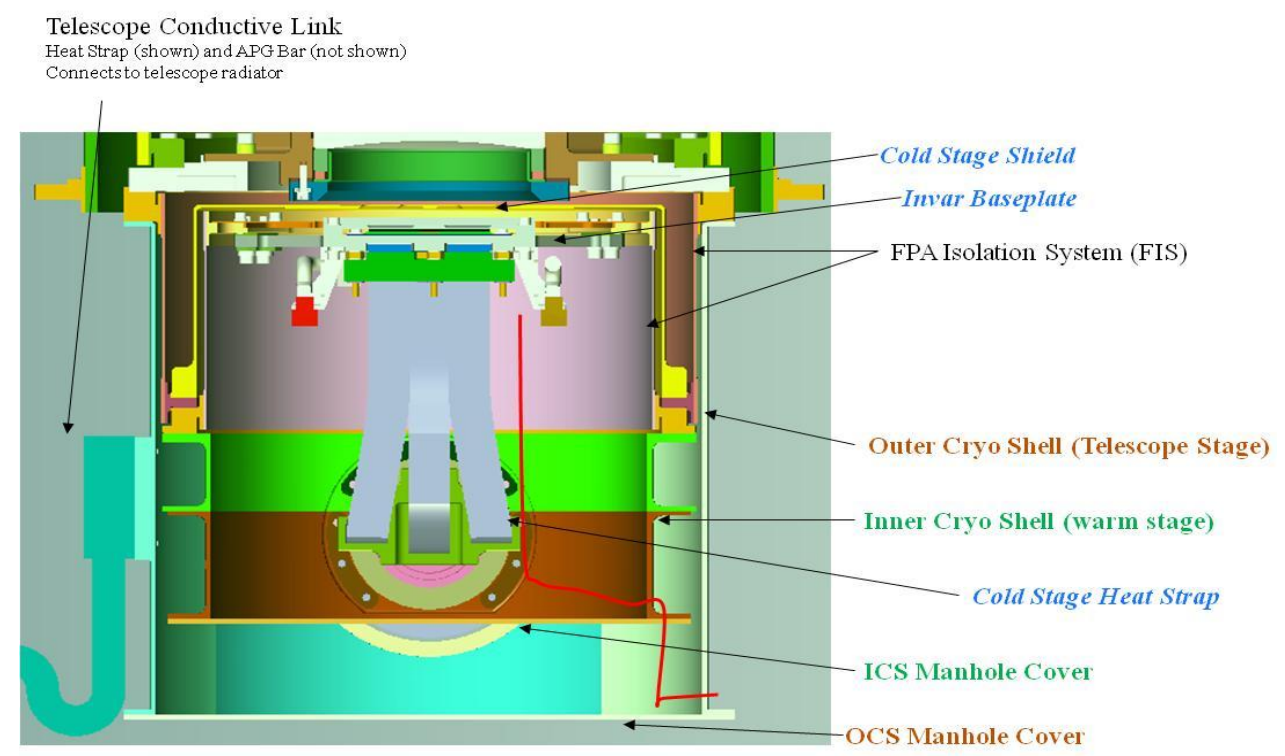

Figure 5. TIRS Cryo Subsystem Design. To minimize heat parasitics to the cold stage a series of Isolation Systems are used.

\section{Telescope Subsystem}

\section{A. Overview of Subsystem}

The telescope subsystem is comprised of the Optical Assembly (TIRS telescope), TIS, Outer Cryo Shell, telescope conductive link (including a FCL and APG bar), and dedicated radiator. Since the Telescope Subsystem is passively cooled, a significant amount of time was spent minimizing potential heat leaks from the warmer surroundings. Use of a complex set of cryogenic MLI blankets that interweave with one another allowed for proper radiative isolation, primarily from the spacecraft deck and TIRS structure. The conductive isolation method used was similar to that of the FPA Isolation System.

The Optical Assembly consists of three Ge lenses and a ZnSe lens, all mounted inside of a three-piece aluminum barrel. Note that an early trade study showed the use of aluminum in reducing thermal gradients outweighed the mechanical benefits of using titanium. The FWD section is closest to the warmer end of the Sensor Unit and holds lenses $1 \& 2$, as well as a set of Operational heaters (OP 4A/B). The AFT section is closest to the colder end of the Sensor Unit (QWIP detectors) and holds lenses $3 \& 4$, as well as a distinct set of Operational heaters (OP 3A/B). The mid-section serves to connect the FWD and AFT sections. The focus of the optical assembly is adjusted via temperature setpoints of the FWD and AFT barrels; nominal settings are $189 \mathrm{~K}$ and $186.5 \mathrm{~K}$ respectively. There is an aluminum low emissivity radiation shield that minimizes interaction between the telescope barrel covered with 1 layer VDA/Kapton blanket and warmer instrument surroundings. Thermal conductive leaks through sensor and heater leads are minimized by the use of constantan FPWs.

The Telescope Isolation System (TIS) uses a similar concept to that of the FIS; it consists of two thin-walled G10 cylinders that are epoxied to aluminum mounting rings. The cold-end aluminum ring is tied to the FIS, while the warm-end aluminum ring is mounted to the TIRS structure, specifically the optical deck. Similar to the FIS, the radiative parasitics are picked off with a shield above and a shell below. The difference is that the gold plated telescope shield joins at the fold ring middle while the Outer Cryo Shell joins at the cold end of the TIS. The Outer Cryo Shell is made of high conductive aluminum 1100 and serves to (1) protect the warm stage Inner Cryo Shell radiatively from the warmer instrument surroundings and (2) provide a conductive path to a dedicated radiator. It also mounts a set of Operational heaters (OP 2A/B) that may be used in conjunction with the Telescope heaters, but are not expected to be needed on orbit during nominal operations. The Outer Cryo Shell is the primary path for the excess telescope heat to make its way to a dedicated telescope radiator via the Telescope Link, which features a high purity aluminum FCL and an APG (Annealed Pyrolytic Graphite) bar. The telescope link is bolted on one end to the 
Outer Cryo Shell and on the other end directly to the telescope radiator heat pipes; all interfaces use indium foil to fill in gaps and improve thermal conductivity.

The telescope radiator is a 75" x 12.45 " aluminum honeycomb design, with embedded dual-bore ethane heat pipes used to spread the heat from the telescope link interface. This radiator was positioned closest to the Earthshield to better protect it from the radiation environment. The front face of the radiator is coated with AZ93 White Paint, while the back is protected with cryogenic MLI blanketing.

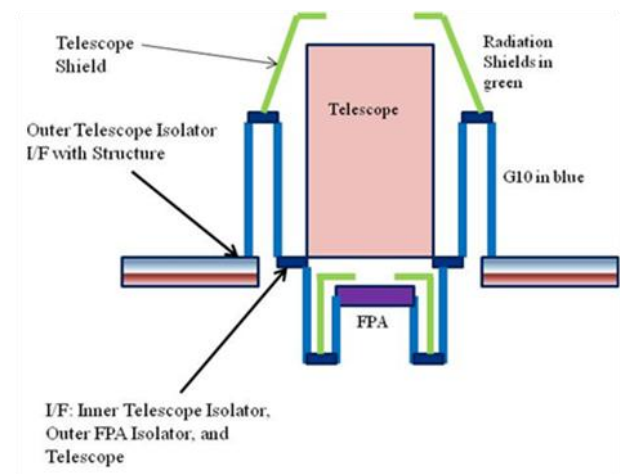

a) TIS and Telescope Radiation Shield

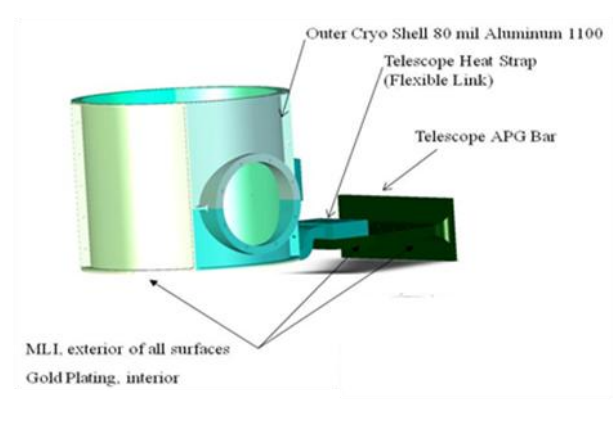

b) Outer Cryo Shell and Telescope Link

Figure 6. TIRS Telescope Subsystem.

\section{B. Design Drivers and Lessons Learned}

Primary Design Driver: Passively cool the optical assembly via a dedicated radiator, while controlling stability to $+/-0.1 \mathrm{~K}(35 \mathrm{sec})$.

Lesson Learned: Material selection requires careful trade studies. A trade study of strength vs. conductivity for various isolator materials was performed before selecting the proper isolators. Trade studies to select a proper type of aluminum were also performed as the effect of material purity on conduction is enhanced at cryogenic temperatures. The original AL-6063 chosen for the Outer Cryo Shell was not available in the desired thickness so a more pure AL-1100 was selected. A trade study was also performed in selecting a method of connection between the Outer Cryo Shell and telescope radiator. The use of APG material proved to be ideal since this material has a sweet spot around $150 \mathrm{~K}$, providing more conduction for less mass and volume compared to any other material. Finally, a trade study was performed for selection of the telescope barrel material; using aluminum over titanium greatly reduced the thermal gradients while maintaining mechanical requirements.

Lesson Learned: Controlling wire parasitics in cryogenic systems is essential. Use of a low conduction metal (i.e. constantan) in thin cross sectional wires (FPW) provides a thermal break between the copper sensor and heater leads and their supply wires. Although this was excellent way to reduce parasitic heat leaks into the system, there were a couple of factors that should be noted; 1) Sensor resistance is increased and the extra resistance of the leads must be accounted for in the calibration (for 2-wire devices only), 2) The resistance in the heater leads causes a voltage drop before the heater resulting in less available heater power, so drop should be taken into account when sizing heaters.

Lesson Learned: Cryogenic MLI blankets need special attention. Begin design/fabrication of blankets (especially cryogenic ones) early; Use of 3D plastic prototypes are very useful for templating, while flight hardware is unavailable; It is critical to ensure there is sufficient clearance in design for blanketing, especially in cryogenic areas where blanketing may be more "puffy".

Lesson Learned: Design with testing in mind. Use of APG bar in design avoided having to use heat pipes to transfer heat from the Telescope to the radiator. In addition, the cryocooler radiator had accommodations for bolting a GSE heat strap as needed during tvac testing.

Lesson Learned: Early procurement of long-lead items. Given a very compressed schedule, it was necessary to begin the competitive procurement process for ethane heat pipes prior to instrument CDR. The disadvantage was the design limitations driven by such early procurements. However, the project opted to buy valuable time by allowing the radiator heat pipe integration to be performed internally at GSFC. 


\section{Scene Select Mechanism}

\section{Overview of Subsystem}

The Scene Select Mechanism (SSM) is comprised of the following components:

- Remote Electronics

- Motor Encoder

- Bearing Housing

- Fixed Baffle

- Rotating Baffle

- Mirror

The SSM Subsystem is mounted to the structure at the bearing housing flanges via a support cylinder called SCONE. The bearing housing (and indirectly the encoder and remote electronics) is controlled via Operational heaters (OP $5 \mathrm{~A} / \mathrm{B}$ ) to within a temperature range of 0 to $+20 \mathrm{C}$, nominal setpoint at $5 \mathrm{C}$. To protect the bearing housing, encoder, and remote electronics during non-operational modes a set of thermostatically controlled survival heaters are mounted to the bearing housing.

The Mirror, which is a highly polished gold plated aluminum surface, is attached to the bearing housing through a titanium shaft, and can rotate to three distinct positions during instrument calibration sequences: the nadir aperture for science data collection, the space aperture for a deep space calibration, and the onboard Blackbody Calibrator for a warmer calibration. The rotating baffle moves with the mirror thus allowing view to only one aperture at a time. The fixed baffle has three large aperture holes. To minimizing stray light most surfaces in this area are black anodize. The decontamination heaters, located on the fixed baffle, are no longer needed for instrument dry-out and de-ice operations, but are instead autonomously commanded to turn on (side B nominally) during the spacecraft safehold mode (specifically the Sun Acquisition Mission Mode). This set of heaters will keep the SSM components warm during spacecraft off-nominal orientations where the environment is cold for the two instruments.

A variety of flight sensors are used to track the SSM component temperatures, including spacecraft-read sensors, heater control sensors, and housekeeping sensors. An additional twenty four (12 A/B) MCE sensors are spread out among the various SSM components; though these can only be read when the MEB/MCE card is powered ON, and only one side can be read at any given time.

\section{Design Drivers and Lessons Learned}

Primary Design Driver: Maintain the SSM within temperature range to allow for proper mirror positioning while minimizing parasitics into the telescope subsystem.

Lesson Learned: Avoid use of low conductivity materials given temperature gradient requirements. The procurement schedule for the SSM bearing housing led to an early selection of titanium material, which resulted in complications during the thermal control system design. In addition, modeling of this area to adequately simulate temperature gradients was difficult without using a significant amount of nodes; the reduced thermal model gradient representations showed differences with that of the detailed thermal model.

Lesson Learned: Be Creative in Solving Issues. The decontamination heater, which was originally intended for instrument dry-out and de-ice activities, was a great solution to new spacecraft safehold maneuvers presented by the program office late in the flow. Use of this heater during these cold environments avoided having to implement new survival heaters or re-test hardware to colder predicted temperatures.

Lesson Learned: Interface Control Documentation should be complete. The TIRS-to-SC ICD should have included specific orbit/SC maneuver definitions for analysis. Both instrument vendors had to make flight hardware changes after instrument deliveries as a result of added analysis cases late in the flow.

\section{Blackbody Calibrator}

\section{A. Overview of subsystem}


The Blackbody Calibrator aboard the TIRS instrument serves as one of two full-aperture calibration sources; the science team will also make use of the instrument space view available. The Blackbody Calibrator consists of an aluminum 6061 target coated in Z307 black paint, an aluminum conductor bar, and a dedicated radiator with Silver Teflon tape to reject excess heat.

There are multiple operational heaters used to control the target to the temperature range of $270 \mathrm{~K}$ to $320 \mathrm{~K}$ (goal of $260 \mathrm{~K}$ to $330 \mathrm{~K}$ as environment permits). The first couple of heaters $(\mathrm{OP} 7 \mathrm{~A} / \mathrm{B})$ are located closest to the target along the aluminum rod. These are used to maintain a stable temperature over the lower half of the temperature range. Two supplemental heaters (OP $8 \&$ 9), located further from the target on the aluminum rod and back of radiator, are used as needed for maintaining temperature over the warmer half of the range. The supplemental heaters are typically used in open loop mode to allow OP $7 \mathrm{~A} / \mathrm{B}$ heaters to

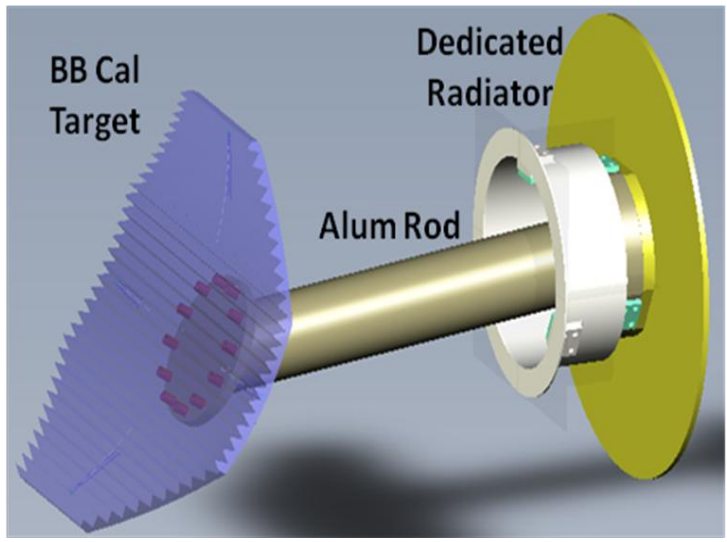

Figure 8. TIRS Blackbody Calibrator. The Blackbody Calibrator is composed of a target, rod, and dedicated radiator. maintain target temperature stability.

To properly isolate the Blackbody subsystem from the surrounding environment, the back of the target and radiator as well as the aluminum rod are covered with MLI blanketing. The Blackbody Calibrator is conductively isolated through 3 thin titanium flexures.

\section{B. Design Drivers and Lessons Learned}

Primary Design Driver: Provide a stable and known temperature within the desired range for science to calibrate instrument.

Lesson Learned: Design for flexibility. For the majority of the instrument life on orbit, the Blackbody Calibrator will be maintained at a nominal temperature of $290 \mathrm{~K}$. The science team will vary the calibrator temperature over a small range during certain periods in the mission, and less frequently over the full specified range. Thermal subsystem had to design for the full temperature range; however, implementing a design capable of controlling different variations of the multiple heaters in order to accommodate seasons and target temperatures gives the instrument flexibility.

\section{Cryocooler Subsystem}

\section{A. Overview of Subsystem}

The cryocooler subsystem consists of a cryocooler ThermoMechanical Unit (TMU), a CryoCooler Mount (CCM), transport ammonia heat pipes, and a dedicated radiator. The cryocooler electronics are located separately on a spacecraft shear panel. The CCM is a highly conductive bracket with embedded APG material that transfers heat from the TMU to ammonia transport heat pipes. The heat pipes then move this heat over to the radiator, where it is transferred via an APG spreader to a set of radiator spreader heat pipes. When the CC is not operating survival heaters mounted to the CCM are necessary to keep the TMU and ammonia heat pipes from freezing. Two thermostatically controlled heater circuits with multiple heater elements wired in parallel are used to keep the CCM above survival limits.

The cryocooler subsystem is responsible for removing heat from the warm and cold stage cold fingers and transferring it to a dedicated radiator on the $+Y$ side of the spacecraft. The inefficient nature of the cryocooler, dissipating 80 Watts for each Watt of power removed from the cold stage, highlights the importance of keeping parasitic heat loads as low as possible. The cryocooler radiator was sized in accordance with pre-CDR project requirements, which held a significant amount of margin to account for unknowns in cryocooler performance capabilities.

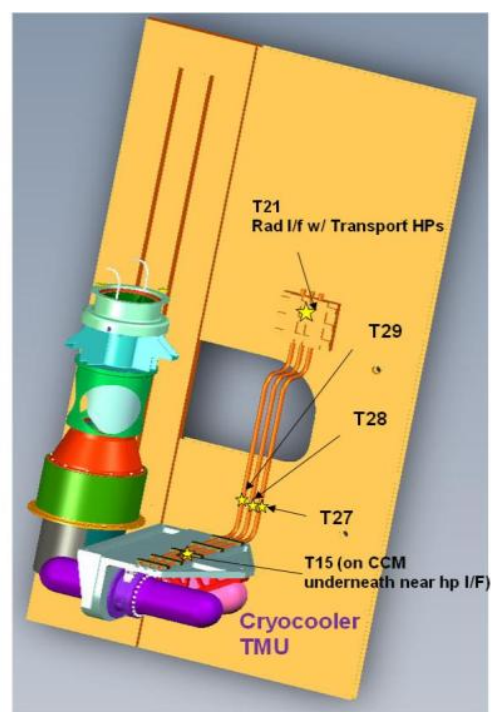

Figure 9. TIRS Cryocooler Subsystem. The TIRS cryocooler subsystem includes CC TMU, CCM, transport heat pipes, and a dedicated radiator. 
After a very successful Cryo Subsystem (CSS) ETU tvac test the parasitic heat loads into the cold and warm stage cold tips were better characterized and so the project was able to more accurately predict Cryocooler power dissipations. Nonetheless, the project decided to maintain a significant amount of margin thru instrument integration and testing. In the end, the cryocooler power dissipations were less than half of the baselined requirements, needing the cryocooler radiator to be partially covered with MLI blanketing.

The cryocooler TMU is in a "stowed" configuration during launch, and is deployed about a week into the dry-out phase of the TIRS instrument. An added feature to the cryocooler TMU mount was that of a means to isolate cryocooler vibrations from the optical deck by using "springy" circle flexures. The flexures, when deployed, are able to minimize the effects of vibration on the optical deck. A Cryocooler Launch Lock is used to "stow" these flexures during launch to prevent possible damage from launch loads experienced during this phase of the mission. Similar to the Earthshield deployment system, the launch lock deployment system has a release mechanism and potentiometer (located external to the primary structure) and internal linkages mounted to a triangular support that is attached to the Cryocooler Mount. The launch lock has a passive thermal design, and is to be maintained within temperature limits pre-deployment.

\section{B. Design Drivers and Lessons Learned}

Primary Design Driver: Provide a highly conductive path to a dedicated radiator in order to remove a significant amount of excess heat during operational modes, while providing a means of safeguarding the hardware when in non-operational modes.

Lesson Learned: Keep it simple. Trade studies were conducted early in the design phase to select a method of transporting heat from the cryocooler; options included (1) simple CCHPs with survival heaters, (2) VCHPs, and (3) loop heat pipes. Although loop heat pipes and VCHPs provide savings in heater power during cryocooler nonoperational modes since the radiator can effectively be shut off when not in use, the TIRS schedule led the thermal team to select a simpler more reliable option, that of CCHPs. A future application that may be power constrained and have more time and money may use a different solution.

Lesson Learned: Use of fancy materials over heat pipes in certain circumstances. There were two primary bottlenecks identified early in the design of the source-to-sink path; these were the Cryocooler Mount (CCM) and the radiator saddle. Design of both areas was greatly simplified with the use of APG material embedded within the aluminum parts to more adequately transfer large amounts of heat to the dedicated radiator.

\section{Structure and Earthshield}

\section{A. Structure}

1. Overview of component

The TIRS structure design features composite facesheets with an aluminum honeycomb. The structure holds all TIRS Sensor Unit components, including internal subsystems like the SSM and CSS and external subsystems like the FPE and Earthshield. The structure is divided into several panels, all bonded together via titanium fittings (except removable panels which are bolted together). The optical deck is the interface to the Optical Assembly, and as such has tighter temperature requirements. A detailed STOP analysis was performed to predict any thermal disturbances to the TIRS line of sight; the optical deck was of particular interest in this analysis. The entire structure is covered with MLI blanketing (kapton outer layer) to protect it and the internal Sensor Unit components from the space environment.

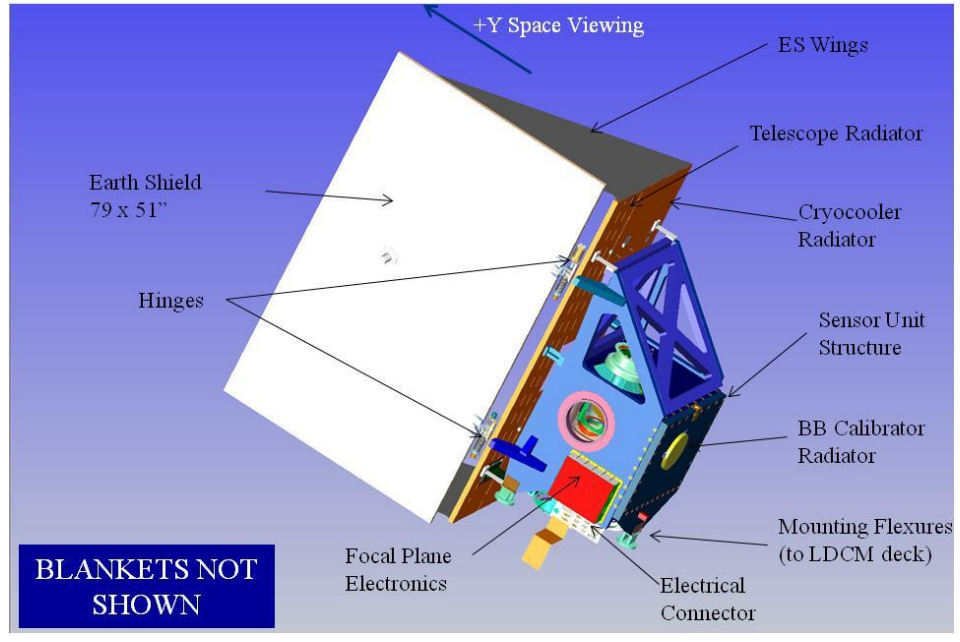

Figure 10. TIRS Structure and Earthshield. The TIRS structure holds all Sensor Unit components, including the large Earthshield. 


\section{Design Drivers and Lessons Learned}

Primary Design Driver: Maintain structure within limits by providing good isolation to space environment and cryogenic components (i.e. telescope radiator mounts directly to structure); maintain structure within pre-launch limits during ground testing.

Lesson Learned: Understand implications of design compromises. The composite structure poor conductance resulted in large temperature gradients and made it difficult to accurately model and predict on-orbit temperatures.

Lesson Learned: Aggressive schedule had requirements before analysis completion. The structural requirements were based on thermal analyses instead of mechanical stress analyses, resulting in two sets of limits (pre and post launch).

Lesson Learned: Have a generous spare philosophy if possible. A 100\% spare policy was used on TIRS thermal hardware, rather than a more typical 50\% philosophy, primarily due to the compressed TIRS schedule. Most thermal hardware procurements, including sensors, heaters, and thermostats, took months to complete, leaving no room for possible design changes post CDR. There were several instances during the development phase when spare parts were used to accommodate changes, such as the science team's request to add sensors on the structure apertures to better understand background noise.

\section{B. Earthshield}

\section{Overview of component}

The TIRS Earthshield serves to protect the telescope and cryocooler $(+\mathrm{Y})$ radiators from the space environment. The Earthshield is 78 " x 50.5" and is made of an aluminum honeycomb with composite facesheets. The Earthshield launches in a stowed configuration, and is deployed after the instrument on-orbit dry-out phase is completed, at approximately L+23 days. This component is held to the primary structure via two hinges, which are mounted off the structure's two outriggers. There is a potentiometer located on the upper hinge, which has a dedicated survival heater to maintain component above limits prior to Earthshield deployment. There is a damper located on the lower hinge, which also has a dedicated survival heater to be used pre-deployment, and also has a deployment heater to maintain the damper within Op limits during Earthshield deploy. Post Earthshield deployment there are no temperature requirements on the potentiometer and damper.

\section{Design Drivers and Lessons Learned}

Primary Design Driver: Provide a specular shield to protect the telescope and cryocooler radiators from the Earth and Solar heat loads, while isolating large temperature fluctuations from the remaining TIRS Sensor Unit.

Lesson Learned: Use clever manufacturing options where possible. The Earthshield VDA layer was co-cured into the layup and thus did not have to be applied after assembly, saving an integration step. This also resulted in a very flat and very specular surface, providing a high quality thermal surface compared to that of other post-cure options available.

Lesson Learned: Training of ALL technicians/engineers/QA on critical surfaces and delicate items. Despite emails and signs posted around the instrument hardware, improper care of delicate surfaces was noted during integration and testing. Proper training should be set in place by projects to ensure all personnel are aware of sensitive thermal surfaces, such as the Earthshield VDA and Z93C55 White Paint surfaces.

\section{Electronics Boxes}

There are three electronics boxes for the TIRS Instrument, not including SSM electronics. The FPE is mounted to the Sensor Unit, and the MEB and CCE are mounted to the spacecraft bus shear panel.

\section{A. Focal Plane Electronics (FPE)}

3. Overview of component

The FPE box is located on the nadir side of the instrument, mounted externally to the structure via four Ti flexures to maintain isolation from the structure. Operational heaters $(\mathrm{OP} 6 \mathrm{~A} / \mathrm{B})$ are located externally on the sides of the box, underneath MLI blanketing. The FPE can be set within a temperature range of $253 \mathrm{~K}$ and $293 \mathrm{~K}$ during the mission life, allowing for flexibility over seasonal variations. However, the nominal setpoint baselined for the mission is $283 \mathrm{~K}$. A series of Flexible Printed Wire (FPW) harnesses are used to connect the FPA to the FPE. FPWs are made of $\mathrm{Cu}$ and constantan to minimize conductive heat to the FPAs. In addition, thermostatically controlled survival heaters are located on the external surface of the FPE baseplate to maintain box survival temperatures when powered OFF.

The FPW provided a means of electrically connecting the FPA to the FPE while keeping the thermal parasitics as low as possible. The cross sectional areas of the traces were reduced as much as possible while keeping the 
integrity of signals and power. Traces were adjusted wider for power transmission and narrower for signals. Use of constantan provided better thermal isolation than copper, though the latter was used between the warm stage and telescope stage to reduce schedule and cost. The constantan FPWs were also used between telescope and room temperature structure to carry heater power and sensor signals.

4. Design Drivers and Lessons Learned

Design driver: Maintain a stable temperature environment while providing good isolation to the FPA and structure.

Lesson Learned: Identify areas of technology development early. A dedicated harness lead was brought on the team for development of FPWs. Thermal team worked very closely with harness lead during design phase to ensure minimum conduction leaks into FPAs from the FPE.

\section{B. Main Electronics Box (MEB) and Cryocooler Electronics (CCE)}

1. Overview of boxes

The MEB and CCE are wet mounted to a shear panel on the spacecraft bus using RTV-566. Externally, the MEB top is covered with Silver Teflon tape for use as a radiator surface to reject excess heat from the internal electronics boards. Three CCE sides are similarly covered with Silver Teflon tape and used as radiator surfaces. The remaining sides of the two electronics boxes are covered with MLI blanketing, including the various harnesses that connect the boxes to the TIRS Sensor Unit and the Spacecraft. The boxes are each thermally controlled by the spacecraft; the external thermal design of the boxes, mainly radiator sizing and blanketing, is the responsibility of the spacecraft vendor, Orbital Sciences Corporation in Phoenix, AZ. The spacecraft thermal design uses survival heaters mounted to the shear panel directly underneath the boxes to protect the hardware from reaching low survival limits.

2. Design Drivers and Lessons Learned

Design Driver: Provide a means to reject excess heat from a high powered box. Spacecraft thermal design uses box sides as radiators and spacecraft shear panel for rejecting excess heat; note thickness of shear panel was increased to better transfer heat from base of boxes to shear panel radiator surface areas.

Lesson Learned: Define power requirements early. MEB and CCE power dissipation values were not well defined early in the design phase. This resulted in numerous interface design iterations with the spacecraft vendor. The primary reasons for lack of this information are (1) the MEB underwent a significant redesign just prior to instrument CDR (2) the CCE power which varies as a function of TMU motor power required adequate subsystem testing.

Lesson Learned: Grounding requirements need to be established early. Lack of clarity with the program grounding requirements resulted in multiple hardware changes late in the test flow. One example had to do with the type of Silver Teflon tape used for radiating surfaces.

Lesson Learned: Standardize component names and mnemonics. Different subsystems refer to the same parts differently. This should be avoided early in the design phase to save time/work and possible confusions later in the development phase. All subsystems need to be involved so that an agreement is made for each heater, sensor, and thermostat to have a unique name (i.e. HS3A-2 or T_TC_BB_CTRL_A_T).

\section{Additional Lessons Learned}

The TIRS thermal team had great communication and synergy, and a lot of good humor was used to reduce stress levels. This was particularly important given the very aggressive TIRS schedule, which resulted in a significant amount of work, continuously. Here are some of the lessons learned that would fall into the "teamwork" category.

Lesson Learned: Divide and conquer. Assign primary core team member to attend meetings or spearhead issues.

Lesson Learned: Make use of team member strengths. Some successful examples include the subsystem procurements, organization, hardware, WOAs, test planning, and procedures.

Lesson Learned: 2+ Back-Up. Always have two core team members involved in all aspects of hardware, testing, requirements, and issues. Other members may also be cognizant and became "second" person as needed.

Lesson Learned: Define and pass. Use non-core team members to execute certain tasks, such as componentlevel testing and creating documentation, in order to alleviate workload from core team.

Lesson Learned: Communicate. Communication within the thermal team, from subsystem to subsystem, and from subsystems to management is critical. 